\title{
Global Existence of Periodic Solutions in a Nonlinear Delay-Coupling Chaos System
}

\author{
Yanqiu Li' ${ }^{1}$, Jihua Yang2 ${ }^{2}$ Feng Rao ${ }^{1}$ \\ ${ }^{1}$ College of Sciences, Nanjing University of Technology, Nanjing, China \\ ${ }^{2}$ Department of Mathematics and Computer Science, Ningxia Normal University, Guyuan, China \\ Email: liyanqiu_1111@163.com, raofeng2002@163.com,jihua1113@163.com
}

Received 29 January 2016; accepted 18 March 2016; published 21 March 2016

Copyright (C) 2016 by authors and Scientific Research Publishing Inc.

This work is licensed under the Creative Commons Attribution International License (CC BY).

http://creativecommons.org/licenses/by/4.0/

(c) ()

\begin{abstract}
The dynamics of a unidirectional nonlinear delayed-coupling chaos system is investigated. Based on the local Hopf bifurcation at the zero equilibrium, we prove the global existence of periodic solutions using a global Hopf bifurcation result due to $\mathrm{Wu}$ and a Bendixson's criterion for higher dimensional ordinary differential equations due to Li \& Muldowney.
\end{abstract}

\section{Keywords}

Unidirectional Delayed-Coupling, Chaos System, Hopf Bifurcation, Periodic Solution

\section{Introduction}

In the 19th century, $\mathrm{H}$. Poincaré found that three-body gravitational interactions can produce amazing complex behaviors by studying the celestial mechanics, that is, there may be uncertainty even in the dynamic equations of very simple object interactions. He found that some systems have sensitive dependence on initial values and behavioral unpredictability. It is the first discovery of chaos. In 1963, E.N. Lorenz [1] unexpectedly discovered the first chaotic attractor in simulating weather, since then, chaos occurs in many areas and has access to the farreaching development.

Since the discovery of chaos, it has been highly regarded in many areas, such as mathematics, mechanics, meteorology, astronomy, and economics. Chaos can be used to achieve the encrypted transmission of information. If the information is hidden in the chaotic signal, when the receiver has synchronized with the transmitter signal, the signal can be obtained, rather than by other people. The important feature of chaos is its highly sensitive to initial values, which makes it difficult to control. In practical applications, we hope to eliminate the negative effects resulting chaos and strengthen its positive effects. This makes the chaos control has become a highly anticipated new field. In particular, we can control the bifurcation of system [2], such as retarding the 
occurrence of inherent bifurcation, stabling bifurcation solution, changing the shape or type of bifurcation, and controlling multiplicity of the limit cycle, amplitude or frequency. It has formed a number of chaos control methods, such as the OGY method [3], variational parameter control [4], state feedback control [5], adaptive control [6], optimal control [7], robust control [8] and non-feedback control [9]. As an important research aspect of chaos control, chaos synchronization has also been widely concerned, resulting in a variety of effective methods: PC synchronization [10], active-passive synchronization [11], chaos synchronization based on mutual coupling [12] and adaptive synchronization method [13]. Chaos control and chaos synchronization are identical. These methods often make the dimension of original system increases, forming a new coupled system. In order to understand the ultimate effect of chaos control and synchronization, we not only need to know the dynamic behavior of original system, but also need to discuss the one of new coupled system (see [14]-[18]).

A system with unidirectional nonlinear delayed-coupling scheme is considered in this paper. T. Banerjee et al. [19] proposed system

$$
\dot{x}=-a x(t)-b f(x(t-\tau))
$$

where $x$ is the state variable, $a, b>0$ are system parameters, and $\tau>0$ is the time delay. $f$ is the nonlinear function. When $f\left(x_{\tau}\right)=-0.5 n\left(\left|x_{\tau}\right|+x_{\tau}\right)+m \tanh \left(l x_{\tau}\right), n, m, l>0$, [19] has reported that as $b$ or $\tau$ varies, chaos and hyperchaos are observed. Furthermore, [20] studied the synchronization of the following coupled system.

$$
\left\{\begin{array}{l}
\dot{x}=-a x(t)-b_{x} f\left(x\left(t-\tau_{1}\right)\right), \\
\dot{y}=-a y(t)-b_{y} f\left(y\left(t-\tau_{1}\right)\right)-b_{c} f\left(x\left(t-\tau_{2}\right)\right),
\end{array}\right.
$$

where $x$ and $y$ are drive and response variables. $\tau_{1}>0$ is the system delay, and $\tau_{2}>0$ is the coupling delay. $b_{x}$ and $b_{y}$ are as usual positive parameters. The value of $b_{c}$ determines the strength of the coupling. Our purpose is to investigate the global existence of periodic solutions for the system.

The remainder of this paper is organized as follows. In Section 2, we employ the preliminary results about the existence of the local Hopf bifurcation. In Section 3, the global Hopf bifurcation is established. An example is given in order to illustrate the results obtained in Section 4.

\section{Preliminary Results}

We present some preliminary results of system (2) about the existence of local periodic solutions. This is the basis of the global Hopf bifurcation.

Let $u_{1}(t)=x\left(t-\tau_{2}\right), u_{2}(t)=y(t)$, and denote $\tau_{1}$ as $\tau$. Using $x$ and $y$ to represent the variables still, Equation (2) can be written into the following system

$$
\left\{\begin{array}{l}
\dot{x}=-a x(t)-b_{x} f(x(t-\tau)) \\
\dot{y}=-a y(t)-b_{y} f(y(t-\tau))-b_{c} f(x(t)) .
\end{array}\right.
$$

Clearly, $(0,0)$ is an equilibrium point. The characteristic equation of its corresponding linear system around $(0,0)$ is

$$
\left(\lambda+a+b_{x} f^{\prime}(0) \mathrm{e}^{-\lambda \tau}\right)\left(\lambda+a+b_{y} f^{\prime}(0) \mathrm{e}^{-\lambda \tau}\right)=0
$$

that is,

$$
\lambda+a+b_{x} f^{\prime}(0) \mathrm{e}^{-\lambda \tau}=0
$$

or

$$
\lambda+a+b_{y} f^{\prime}(0) \mathrm{e}^{-\lambda \tau}=0 .
$$

When $\tau=0$, the eigenvalues are $\lambda_{1}=-\left(a+b_{x} f^{\prime}(0)\right)<0, \lambda_{2}=-\left(a+b_{y} f^{\prime}(0)\right)<0$.

Let $\pm \mathrm{i} \omega(\omega>0)$ be a pair of roots of Equation (5). Substitute $\mathrm{i} \omega$ into Equation (5) and separate the real and imaginary parts 


$$
\left\{\begin{array}{l}
a+b_{x} f^{\prime}(0) \cos \omega \tau=0, \\
\omega-b_{x} f^{\prime}(0) \sin \omega \tau=0, k=0,1,2, \cdots .
\end{array}\right.
$$

Denote $\left(H_{1}\right) \quad b_{x}^{2} f^{\prime 2}(0)-a^{2}>0$ and $\left(H_{2}\right) \quad b_{y}^{2} f^{\prime 2}(0)-a^{2}>0$. Let $\lambda(\tau)=\alpha(\tau)+\mathrm{i} \omega(\tau)$ be a root of Equation (4) near $\tau=\tau_{k}(j)$ satisfying $\alpha\left(\tau_{k}(j)\right)=0, \omega\left(\tau_{k}(j)\right)=\omega_{k}, j=0,1,2, \cdots, k=1,2$.

Lemma 1. If $\left(H_{1}\right)$ or $\left(H_{2}\right)$ is satisfied, then

$$
\operatorname{Re}\left(\left.\frac{\mathrm{d} \lambda}{\mathrm{d} \tau}\right|_{\lambda=\mathrm{i} \omega_{k}, \tau=\tau_{k}(j)}\right)>0 .
$$

Lemma 2. 1) If $\left(H_{1}\right)$ and $\left(H_{2}\right)$ are not satisfied, then all roots of Equation (4) have negative real parts for any $\tau \geq 0$.

2) If $\left(H_{1}\right)$ is satisfied, then there exists a sequence of $\tau_{1}(j)$ satisfying $0<\tau_{1}(0)<\tau_{1}(1)<\cdots<\tau_{1}(n)<\cdots$ such that Equation (4) has a pair of purely imaginary roots $\pm i \omega_{1}$ when $\tau=\tau_{1}(j)$, and all roots of Equation (4) have negative real parts when $\tau \in\left[0, \tau_{1}(0)\right)$.

3) If $\left(\mathrm{H}_{2}\right)$ is satisfied, then there exists a sequence of $\tau_{2}(j)$ satisfying $0<\tau_{2}(0)<\tau_{2} 1<\cdots<\tau_{2}(n)<\cdots$ such that Equation (4) has a pair of purely imaginary roots $\pm \mathrm{i} \omega_{2}$ when $\tau=\tau_{2}(j)$, and all roots of Equation (4) have negative real parts when $\tau \in\left[0, \tau_{2}(0)\right)$.

4) If $\left(H_{1}\right)$ and $\left(H_{2}\right)$ are satisfied, then there exists a sequence of $\tau_{k}(j)$ satisfying

$0<\tau_{1}(0)<\tau_{1}(1)<\cdots<\tau_{1}(n)<\cdots$ and $0<\tau_{2}(0)<\tau_{2}(1)<\cdots<\tau_{2}(n)<\cdots$ such that Equation (4) has two pairs of purely imaginary roots $\pm \mathrm{i} \omega_{k}$ when $\tau=\tau_{k}(j)$, and all roots of Equation (4) have negative real parts when $\tau \in\left[0, \min \left\{\tau_{1}(0), \tau_{2}(0)\right\}\right)$, where

$$
\omega_{1}=\sqrt{b_{x}^{2} f^{\prime 2}(0)-a^{2}}, \tau_{1}(j)=\frac{1}{\omega_{0}}\left(\arccos \frac{a}{b_{x} f^{\prime}(0)}+2 j \pi\right),
$$

and

$$
\omega_{2}=\sqrt{b_{y}^{2} f^{\prime 2}(0)-a^{2}}, \tau_{2}(j)=\frac{1}{\omega_{2}}\left(\arccos \frac{a}{b_{y} f^{\prime}(0)}+2 j \pi\right), j=0,1,2, \cdots .
$$

Using the lemmas above, we have Theorem 1.

Theorem 1. Suppose $\left(H_{1}\right)$ is satisfied.

1) If $\left(H_{1}\right)$ and $\left(H_{2}\right)$ are not satisfied, then the zero equilibrium point of system (3) is asymptotically stable for any $\tau \geq 0$.

2) If $\left(H_{1}\right)$ is satisfied, then the zero equilibrium point of system (3) is asymptotically stable when $\tau \in\left[0, \tau_{1}(0)\right)$ and unstable when $\tau>\tau_{1}(0)$. System (3) undergoes a Hopf bifurcation at when $\tau=\tau_{1}(j)$.

3) If $\left(\mathrm{H}_{2}\right)$ is satisfied, then the zero equilibrium point of system (3) is asymptotically stable when $\tau \in\left[0, \tau_{2}(0)\right)$ and unstable when $\tau>\tau_{2}(0)$. System (3) undergoes a Hopf bifurcation when $\tau=\tau_{2}(j)$.

4) If $\left(H_{1}\right)$ and $\left(H_{2}\right)$ are satisfied, then the zero equilibrium point of system (3) is asymptotically stable when $\tau \in\left[0, \min \left\{\tau_{1}(0), \tau_{2}(0)\right\}\right)$ and unstable when $\tau>\min \left\{\tau_{1}(0), \tau_{2}(0)\right\}$. System (3) undergoes a Hopf bifurcation when $\tau=\tau_{k}(j)$, where $\tau_{k}(j), k=1,2$ are defined above.

\section{Global Existence of Periodic Solutions}

In this section, we study the global continuation of periodic solutions bifurcating from the point $\left(z_{*}, \tau_{k}(j)\right)$, $Z_{*}=(0,0), \quad k=1,2, \quad j=0,1,2, \cdots$. Throughout this section, we follow closely the notations in Wu [21] and let $\left(H_{1}\right)$ or $\left(H_{2}\right)$ be satisfied, namely local Hopf bifurcation occurs. We define

$$
\begin{aligned}
& X=C\left([-\tau, 0], \mathbb{R}^{2}\right), \\
& \Sigma=\operatorname{Cl}\left\{(z, \tau, l):(z, \tau, l) \in X \times \mathbb{R}_{+} \times \mathbb{R}_{+}, z \text { is a } l \text {-periodic solution of system }(2.1)\right\}, \\
& N=\left\{(\hat{z}, \tau, l): \hat{z}=(\hat{x}, \hat{y}),-a \hat{x}=b_{x} f(\hat{x}),-a \hat{y}=b_{y} f(\hat{y})+b_{c} f(\hat{x})\right\}, \\
& \Delta_{\left(z_{*}, \tau, l\right)}(\lambda)=\left(\lambda+a+b_{x} f^{\prime}(0) \mathrm{e}^{-\lambda \tau}\right)\left(\lambda+a+b_{y} f^{\prime}(0) \mathrm{e}^{-\lambda \tau}\right)
\end{aligned}
$$


and let $C\left(z_{*}, \tau_{k}(j), 2 \pi / \omega_{k}\right)$ denote the connected component of $\left(z_{*}, \tau_{k}(j), 2 \pi / \omega_{k}\right)$ in $\Sigma$, where $\omega_{k}$ and $\tau_{k}(j)$ are defined in Lemma 2 .

We assume $\left(H_{1}\right)$ or $\left(H_{2}\right)$ is satisfied so that the local Hopf bifurcation occurs.

Lemma 3. If $f(x)$ is bounded, then all periodic solutions of the system (3) are uniformly bounded.

Proof. Suppose that there exists $L>0$ such that $|f(x)| \leq L(\forall x \in \mathbb{R}),(x(t), y(t))$ is a nonconstant periodic solution of system (3) and $x(t), y(t)$ have maximums at $t_{1}, t_{2}$, respectively, then $x^{\prime}\left(t_{1}\right)=0, y^{\prime}\left(t_{2}\right)=0$. We have

$$
\begin{gathered}
\left|x\left(t_{1}\right)\right|=\frac{b_{x}}{a}\left|f\left(x\left(t_{1}-\tau\right)\right)\right| \leq \frac{b_{x} L}{a}, \\
\left|y\left(t_{2}\right)\right|=\frac{1}{a}\left|b_{y} f\left(y\left(t_{2}-\tau\right)\right)+b_{c} f\left(x\left(t_{2}\right)\right)\right| \leq \frac{L}{a}\left(b_{y}+b_{c}\right) .
\end{gathered}
$$

This shows that the periodic solutions of (3) are uniformly bounded.

Lemma 4. System (3) has no nontrivial $\tau$-periodic solution.

Proof. If system (3) has a nontrivial $\tau$-periodic solution, then

$$
\begin{aligned}
& \dot{x}(t)=-a x(t)-b_{x} f(x(t)), \\
& \dot{y}(t)=-a y(t)-b_{y} f(y(t))-b_{c} f(x(t)),
\end{aligned}
$$

has a nontrivial periodic solution.

However, system (8) only has trivial periodic solutions. In fact,

$$
\dot{x}(t)=-a x(t)-b_{x} f(x(t))
$$

only has a trivial periodic solution $\bar{X}$ (i.e., equilibrium). Moreover,

$$
\dot{y}(t)=-a y(t)-b_{y} f(y(t))-b_{c} f(\bar{x})
$$

has no nontrivial periodic solution.

Thus, system (3) has no nontrivial $\tau$-periodic solution.

Next, we show system (3) has no nontrivial $2 \tau$-periodic solution.

Lemma 5. Assume

$$
\left(H_{3}\right) 2 a>\max \left\{b_{x}\left|f^{\prime}(z)\right|, b_{y}\left|f^{\prime}(z)\right|, b_{c}\left|f^{\prime}(z)\right|\right\}
$$

is satisfied, system (3) has no nontrivial $4 \tau$-periodic solution. Moreover, system (3) has no nontrivial $2 \tau$-periodic solution.

Proof. Let $z(t)=(x(t), y(t))$ be a $4 \tau$-periodic solution of system (3).

$$
u_{k}(t)=\left(x_{k}(t), y_{k}(t)\right) \triangleq z(t-(k-1) \tau), k=1,2,3,4 .
$$

Then $u(t)=\left(u_{1}(t), u_{2}(t), u_{3}(t), u_{4}(t)\right)$ is a periodic solution to the following system of ODE:

$$
\begin{aligned}
& \dot{x}_{i}=-a x_{i}-b_{x} f\left(x_{i+1}\right), \\
& \dot{y}_{i}=-a y_{i}-b_{y} f\left(y_{i+1}\right)-b_{c} f\left(x_{i}\right),
\end{aligned}
$$

where $i=1,2,3,4$ and $x_{5} \triangleq x_{1}, y_{5} \triangleq y_{1}$.

From Lemma 3, the periodic orbit of the system (9) belongs to the region:

$$
G=\left\{u \in \mathbb{R}^{8}|| x_{k}\left|\leq \frac{b_{x} L}{a},\right| y_{k} \mid \leq \frac{L}{a}\left(b_{y}+b_{c}\right), k=1,2,3,4\right\} .
$$

If we want to prove there is no nontrivial $4 \tau$-periodic solution in (3), it suffices to prove that there is no nonconstant periodic solution for (9). To do this, we apply the general Bendixson's criterion in higher dimensions developed by Li \& Muldowney [22]. It is easy to compute the Jacobian matrix $J(u)$ of the system (9) for $u \in \mathbb{R}^{8}$ : 


$$
J(u)=-\left(\begin{array}{cccccccc}
a & 0 & b_{x} f^{\prime}\left(x_{2}\right) & 0 & 0 & 0 & 0 & 0 \\
b_{c} f^{\prime}\left(x_{1}\right) & a & 0 & b_{y} f^{\prime}\left(y_{2}\right) & 0 & 0 & 0 & 0 \\
0 & 0 & a & 0 & b_{x} f^{\prime}\left(x_{3}\right) & 0 & 0 & 0 \\
0 & 0 & b_{c} f^{\prime}\left(x_{2}\right) & a & 0 & b_{y} f^{\prime}\left(y_{3}\right) & 0 & 0 \\
0 & 0 & 0 & 0 & a & 0 & b_{x} f^{\prime}\left(x_{4}\right) & 0 \\
0 & 0 & 0 & 0 & b_{c} f^{\prime}\left(x_{3}\right) & a & 0 & b_{y} f^{\prime}\left(y_{4}\right) \\
b_{x} f^{\prime}\left(x_{1}\right) & 0 & 0 & 0 & 0 & 0 & a & 0 \\
0 & b_{y} f^{\prime}\left(y_{1}\right) & 0 & 0 & 0 & 0 & b_{c} f^{\prime}\left(x_{4}\right) & a
\end{array}\right) .
$$

Then the second additive compound matrix $J^{[2]}(u)$ of $J(u)$ is a $\left(\begin{array}{l}8 \\ 2\end{array}\right) \times\left(\begin{array}{l}8 \\ 2\end{array}\right)$ matrix defined as follows. For any integers $i, j \in N=\{1,2, \cdots, 28\}$, the element in the $i$-row and the $j$-column of $J^{[2]}(u)$ is

$$
b_{i j}= \begin{cases}-2 a, & \text { if } i=j=1,2, \cdots, 28, \\ b_{x} f^{\prime}\left(x_{1}\right), & \text { if }(i, j) \in\{(12,1),(17,2),(21,3),(24,4),(26,5)\}, \\ -b_{x} f^{\prime}\left(x_{1}\right), & \text { if }(i, j) \in\{(28,7)\}, \\ b_{x} f^{\prime}\left(x_{2}\right), & \text { if }(i, j) \in\{(1,8)\}, \\ -b_{x} f^{\prime}\left(x_{2}\right), & \text { if }(i, j) \in\{(3,14),(4,15),(5,16),(6,17),(7,18)\}, \\ b_{x} f^{\prime}\left(x_{3}\right), & \text { if }(i, j) \in\{(2,4),(14,19)\}, \\ -b_{x} f^{\prime}\left(x_{3}\right), & \text { if }(i, j) \in\{(8,10),(14,23),(17,24),(18,25)\}, \\ b_{x} f^{\prime}\left(x_{4}\right), & \text { if }(i, j) \in\{(23,26)\}, \\ -b_{x} f^{\prime}\left(x_{4}\right), & \text { if }(i, j) \in\{(4,6),(10,12),(15,17),(19,21)\}, \\ b_{y} f^{\prime}\left(y_{1}\right), & \text { if }(i, j) \in\{(18,8),(22,9),(26,10),(27,11),(28,12)\}, \\ b_{y} f^{\prime}\left(y_{2}\right), & \text { if }(i, j) \in\{(7,1)\}, \\ -b_{y} f^{\prime}\left(y_{2}\right), & \text { if }(i, j) \in\{(8,14)\}, \\ -b_{y} f^{\prime}\left(y_{3}\right), & \text { if }(i, j) \in\{(3,5),(9,11),(14,16),(19,23),(19,24),(21,26),(22,27)\}, \\ b_{y} f^{\prime}\left(y_{4}\right), & \text { if }(i, j) \in\{(26,28)\}, \\ -b_{y} f^{\prime}\left(y_{4}\right), & \text { if }(i, j) \in\{(5,7),(11,13),(16,18),(20,22),(23,25)\}, \\ -b_{c} f^{\prime}\left(x_{1}\right), & \text { if }(i, j) \in\{(8,2),(9,3),(10,4),(12,5),(12,6),(13,7)\}, \\ -b_{c} f^{\prime}\left(x_{2}\right), & \text { if }(i, j) \in\{(3,2),(9,8),(19,15),(20,16),(21,17),(22,18)\}, \\ -b_{c} f^{\prime}\left(x_{3}\right), & \text { if }(i, j) \in\{(5,4),(11,10),(16,15),(20,19),(26,24),(27,25)\}, \\ -b_{c} f^{\prime}\left(x_{4}\right), & \text { if }(i, j) \in\{(7,6),(13,12),(18,17),(22,21),(25,24),(25,28),(27,26)\}, \\ 0, & \text { others. }\end{cases}
$$

Choose a vector form in $\mathbb{R}^{28}$ as

$$
\left|\left(x_{1}, x_{2}, \cdots, x_{28}\right)\right|=\max \left\{\left|x_{i}\right|\right\}, i=1,2, \cdots, 28 .
$$

With respect to this norm, we can obtain that the Lozinskil measure $\mu\left(J^{[2]}(u)\right)$ of the matrix $J^{[2]}(u)$ is given by

$$
\begin{aligned}
\mu\left(J^{[2]}(u)\right)= & \max \left\{-2 a+b_{x}\left|f^{\prime}\left(z_{0}\right)\right|,-2 a+b_{y}\left|f^{\prime}\left(z_{0}\right)\right|,-2 a+b_{c}\left|f^{\prime}\left(z_{0}\right)\right|\right\}, \\
& z_{0} \in\left\{x_{1}, y_{1}, x_{2}, y_{2}, x_{3}, y_{3}, x_{4}, y_{4}\right\} .
\end{aligned}
$$


By Corollary 3.5 of Li \& Muldowney [22], the system (9) has no periodic orbit in $G$ if $\mu\left(J^{[2]}(u)\right)<0$. By (11), we have $\mu\left(J^{[2]}(u)\right)<0$ if and only if

$$
2 a>\max \left\{b_{x}\left|f^{\prime}(z)\right|, b_{y}\left|f^{\prime}(z)\right|, b_{c}\left|f^{\prime}(z)\right|\right\} .
$$

So we get (9) only has trivial periodic solutions when $\left(H_{2}\right)$ is satisfied.

Thus, (9) has no nontrivial periodic solution. System (3) has no $4 \tau$-periodic solution.

Theorem 2. Suppose that $\left(H_{1}\right) /\left(H_{2}\right)$ and $\left(H_{3}\right)$ are satisfied, then, for each $\tau>\tau_{k}(j)$, system (3) has $j+1$ nonconstant periodic solutions with periods in $\left(\frac{2 \tau}{2 \sigma+1}, \frac{4 \tau}{4 \sigma+1}\right), \sigma=0,1,2, \cdots, j$, respectively. Here, $\tau_{k}(j), k=1,2, j=0,1,2, \cdots$ are defined in Lemma 2.

Proof. We can prove that the projection of $C\left(z_{*}, \tau_{k}(j), 2 \pi / \omega_{k}\right)$ onto $\tau$-space includes $\left[\tau_{k}(j), \infty\right), j \geq 1$. We have given the characteristic matrix of the system (3) at zero equilibrium.

By Lemmas 1 and 2, there exist $\varepsilon>0, \delta>0$ and a smooth curve $\lambda:\left(\tau_{k}(j)-\delta, \tau_{k}(j)+\delta\right) \rightarrow C$ such that $\tau \in\left[\tau_{k}(j)-\delta, \tau_{k}(j)+\delta\right]$ and

$$
\lambda\left(\tau_{k}(j)\right)=\mathrm{i} \omega_{k},\left.\frac{\mathrm{d} \operatorname{Re}(\lambda(\tau))}{\mathrm{d} \tau}\right|_{\tau=\tau_{k}(j)}>0 .
$$

Then $\Delta(\lambda(\tau))=0,\left|\lambda(\tau)-\mathrm{i} \omega_{k}\right|<\varepsilon$.

Denote $l_{p}=2 \pi / \omega_{k}$ and

$$
\Omega_{\varepsilon}=\left\{(u, l): 0<u<\varepsilon,\left|l-l_{p}\right|<\varepsilon\right\} .
$$

Obviously, if $\left|\tau-\tau_{k}(j)\right| \leq \delta$ and $(u, l) \in \partial \Omega_{\varepsilon}$ such that $\Delta_{\left(z_{*}, \tau, l\right)}(u+2 \pi \mathrm{i} / l)=0$, then $\tau=\tau_{k}(j), u=0$, $l=l_{p}$. Set

$$
H^{ \pm}\left(z_{*}, \tau_{k}(j), 2 \pi / \omega_{k}\right)(u, l)=\Delta_{\left(z_{*}, \tau_{k}(j) \pm \delta, l\right)}(u+2 \pi \mathrm{i} / l) .
$$

We obtain the crossing number

$$
\begin{aligned}
\gamma_{1}\left(z_{*}, \tau_{k}(j), 2 \pi / \omega_{k}\right)= & \operatorname{deg}_{B}\left(H^{-}\left(z_{*}, \tau_{k}(j), 2 \pi / \omega_{k}\right), \Omega_{\varepsilon}\right) \\
& -\operatorname{deg}_{B}\left(H^{+}\left(z_{*}, \tau_{k}(j), 2 \pi / \omega_{k}\right), \Omega_{\varepsilon}\right)=-1 .
\end{aligned}
$$

We conclude that

$$
\sum_{(\hat{z}, \tau, l) \in C\left(z_{*}, \tau_{k}(j), 2 \pi / \omega_{k}\right) \cap N} \gamma_{1}(\hat{z}, \tau, l)<0 .
$$

By Theorem 3.3 of $\mathrm{Wu}[21], C\left(z_{*}, \tau_{k}(j), 2 \pi / \omega_{k}\right)$ is unbounded.

Lemma 3 implies that the projection of $C\left(z_{*}, \tau_{k}(j), 2 \pi / \omega_{k}\right)$ onto the $z$-space is bounded.

From the definition of $\tau_{k}(j)$, we know that

$$
\frac{\pi}{2}<\tau_{k}(0) \omega_{k}<\pi,\left(2 j+\frac{1}{2}\right) \pi<\tau_{k}(j) \omega_{k}<(2 j+1) \pi, j \geq 1 .
$$

Hence

$$
2 \tau_{k}(0)<\frac{2 \pi}{\omega_{k}}<4 \tau_{k}(0), \frac{2 \tau_{k}(j)}{2 j+1}<\frac{2 \pi}{\omega_{k}}<\frac{4 \tau_{k}(j)}{4 j+1}, j \geq 1 .
$$

From Lemmas 4 and 5, we know that $2 \tau<l<4 \tau$ if $(z, \tau, l) \in C\left(z_{*}, \tau_{k}(0), 2 \pi / \omega_{k}\right)$, and $2 \tau /(2 j+1)<l<4 \tau /(4 j+1)$ if $(z, \tau, l) \in C\left(z_{*}, \tau_{k}(j), 2 \pi / \omega_{k}\right), j \geq 1$. So, to make $C\left(z_{*}, \tau_{k}(j), 2 \pi / \omega_{k}\right)$ unbounded, the projection of $C\left(z_{*}, \tau_{k}(j), 2 \pi / \omega_{k}\right)$ onto $\tau$-space must be unbounded. Obviously, $C\left(z_{*}, \tau_{k}(j), 2 \pi / \omega_{k}\right)$ are pairwise disjoint. So system (3) has $j+1$ nonconstant periodic solutions for $\tau>\tau_{k}(j)$.

In this section, we derive the global existences, number and periods of periodic solutions. However, the stability of periodic solutions far away from $\tau_{k}(j), k=1,2, j=0,1,2, \cdots$ is unclear. 


\section{An Example}

Choosing $a=1, b_{x}=1.7, b_{y}=0.45, b_{c}=1.25$ and $f\left(x_{\tau}\right)=-0.5 n\left(\left|x_{\tau}\right|+x_{\tau}\right)+m \tanh \left(l x_{\tau}\right)$ with $m=1, n=0.5, l=1.5$, system (2) can be expressed as follows:

$$
\left\{\begin{aligned}
\dot{x}= & -x(t)-1.7\left[-0.25\left(\left|x\left(t-\tau_{1}\right)\right|+x\left(t-\tau_{1}\right)\right)+\tanh \left(1.5 x\left(t-\tau_{1}\right)\right)\right], \\
\dot{y}= & -y(t)-0.45\left[-0.25\left(\left|y\left(t-\tau_{1}\right)\right|+y\left(t-\tau_{1}\right)\right)+\tanh \left(1.5 y\left(t-\tau_{1}\right)\right)\right] \\
& -1.25\left[-0.25\left(\left|x\left(t-\tau_{2}\right)\right|+x\left(t-\tau_{2}\right)\right)+\tanh \left(1.5 x\left(t-\tau_{2}\right)\right)\right] .
\end{aligned}\right.
$$

[19] gave the curves of $f\left(x_{\tau}\right)$ corresponding to different parameters $n, m$ and $l$ (see Figure 1). It evidences $f\left(x_{\tau}\right)$ is bounded when $x_{\tau} \in(-\infty, A)$ ( $A$ is any finite constant).

For system (13), $b_{x}^{2} f^{\prime 2}(0)-a^{2}=1.89>0, b_{y}^{2} f^{\prime 2}(0)-a^{2}=-0.7975<0$ and $\omega_{1}=1.3748$. $\left(H_{1}\right)$ is satisfied and $\left(H_{2}\right)$ isn't. Furthermore, $\tau_{1}=\tau_{1}(0)=0.6851$.

System (13) has a periodic solution near $\tau_{1}(0)$. As shown in Figure 2 at $\tau_{1}=0.7$.

$\left(H_{3}\right)$ is correct, and we now show large amplitude periodic solutions exist for values of $\tau$ far away from $\tau_{1}(0)$. This indicates the global existence of periodic solutions. As shown in Figure 3 at $\tau=6.5$.

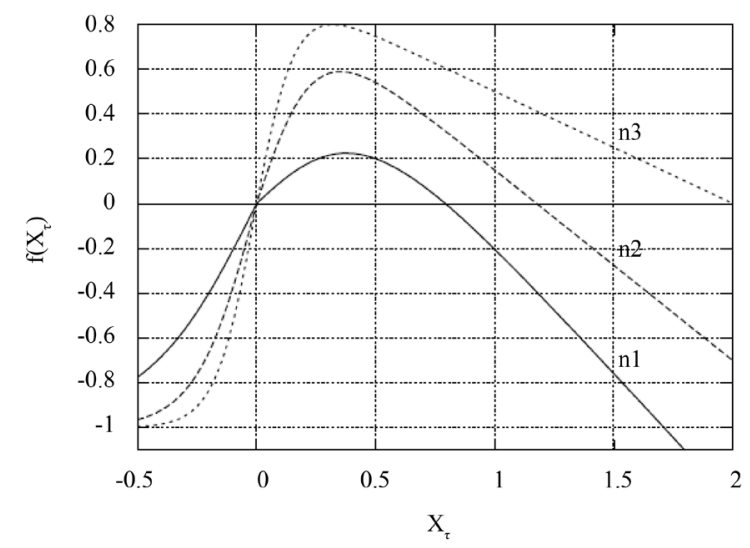

Figure 1. Nonlinearity with the function $f\left(x_{\tau}\right)=-0.5 n\left(\left|x_{\tau}\right|+x_{\tau}\right)+m \tanh \left(l x_{\tau}\right)$ with “n1”: $\quad n=1.15, m=0.97, l=2.19 ; \quad$ "n2”: $\quad n=0.85, m=1, l=4$; “n3”: $n=0.5, m=1, l=5$.
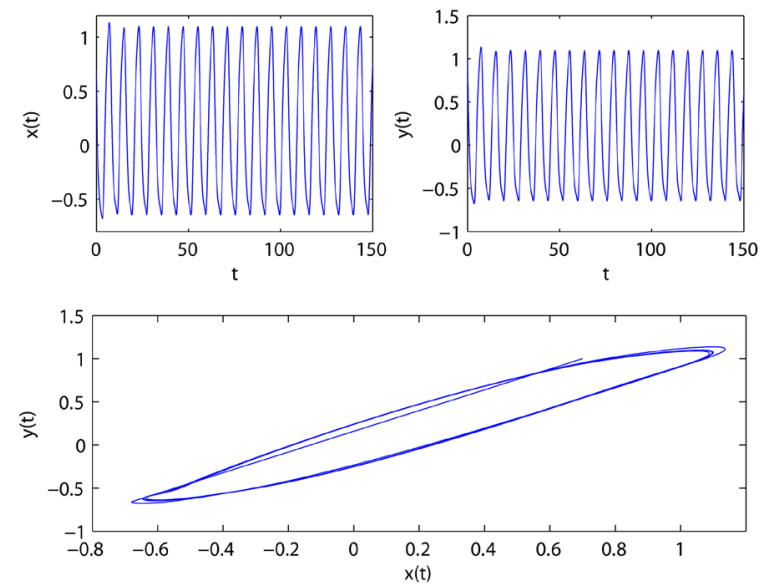

Figure 2. Numerical simulations of a periodic solution to system (13) when $\tau_{1}=0.7$ is near $\tau_{1}(0)=0.6851$. 

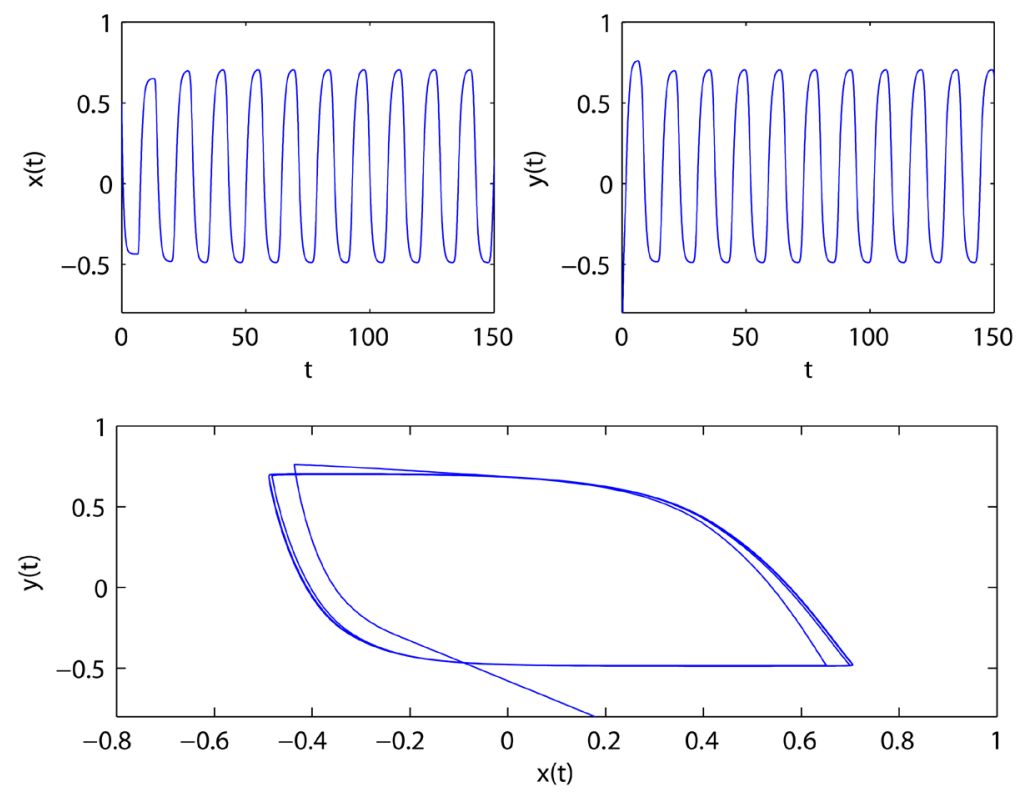

Figure 3. Numerical simulations of a periodic solution to system (13) when $\tau=6.5$ is far away from $\tau_{1}(0)=0.6851$.

\section{Conclusion}

In our paper, the effect of parameters on dynamics of a unidirectional nonlinear delayed-coupling chaos system at the zero fixed point is investigated. There exist the critical values of Hopf bifurcation $\tau_{k}(j), k=1,2, j=0,1,2, \cdots$ and small amplitude periodic solutions. Furthermore, we derive that the local periodic solutions also exist globally for $\tau \geq 0$. In addition, the results indicate the variation of dynamics of system (2) is owing to the inherent delay, and not owing to the coupled one. Our results are propitious to investigate chaos synchronization using system (2), especially synchronization of periodic solutions. However, it still needs to study further for the dynamics of bidirectional coupled system.

\section{Acknowledgements}

We thank the Editor and the referee for their comments. The research is supported by National Natural Science Foundation of China (No. 11301263), the Jiangsu Natural Science Foundation (No. BK20140927), the Ningxia Natural Science Foundation (No. NZ13213) and the Ningxia Higher Educational Science Program (No. GX2014[222]17).

\section{References}

[1] Lorenz, E.N. (1963) Deterministic Non-Periodic Flow. Journal of the Atmospheric Sciences, 20, 130-141. http://dx.doi.org/10.1175/1520-0469(1963)020<0130:DNF>2.0.CO;2

[2] Chen, G., Moiola, J.L. and Wang, H. (2000) Bifurcation Control: Theories, Methods, and Applications. International Journal of Bifurcation and Chaos, 10, 511-548. http://dx.doi.org/10.1142/S0218127400000360

[3] Ott, E., Grebogi, C. and Yorke, J.A. (1990) Controlling Chaos. Physical Review Letters, 64, 1196-1199. http://dx.doi.org/10.1103/PhysRevLett.64.1196

[4] Mondragón, R.J. and Arrowsmith, D.K. (1997) Tracking Unstable Fixed Points in Parametrically Dynamic Systems. Physics Letters A, 229, 88-96. http://dx.doi.org/10.1016/S0375-9601(97)00174-6

[5] Brandt, M.E. and Chen, G. (1996) Feedback Control of a Quadratic Map Model of Cardiac Chaos. International Journal of Bifurcation and Chaos, 6, 715-723. http://dx.doi.org/10.1142/S0218127496000370

[6] Wu, X. and Lu, J. (2004) Adaptive Control of Uncertain Lü System. Chaos Solitons Fractals, 22, 375-381. http://dx.doi.org/10.1016/j.chaos.2004.02.012

[7] Mettin, R. (1998) Control of Chaotic Maps by Optimized Periodic Inputs. International Journal of Bifurcation and 
Chaos, 8, 1707-1711. http://dx.doi.org/10.1142/S0218127498001388

[8] Kwan, C. and Lewis, L. (2000) Robust Backstepping Control of Nonlinear Systems Using Neutral Networks. IEEE Transactions on Systems, Man, and Cybernetics - Part A: Systems and Humans, 30, 753-765.

[9] Belhaq, M. and Houssni, M. (2000) Suppression of Chaos in Averaged Oscillator Driven by External and Parametric Excitations. Chaos Solitons Fractals, 11, 1237-1246. http://dx.doi.org/10.1016/S0960-0779(98)00334-8

[10] Pecora, L.M. and Carroll, T.L. (1990) Synchronization in Chaotic Systems. Physical Review Letters, 64, 821-823. http://dx.doi.org/10.1103/PhysRevLett.64.821

[11] Kocarev, L. and Parlitz, U. (1995) General Approach for Chaotic Synchronization with Applications to Communication. Physical Review Letters, 74, 5028-5031. http://dx.doi.org/10.1103/PhysRevLett.74.5028

[12] Sun, J. and Zhang, Y. (2004) Some Simple Global Synchronization Criterions for Coupled Time-Varying Chaotic Systems. Chaos Solitons Fractals, 1, 93-98. http://dx.doi.org/10.1016/S0960-0779(03)00083-3

[13] Han, X., Lu, J. and Wu, X. (2004) Adaptive Feedback Synchronization of Lü System. Chaos Solitons Fractals, 22, 221-227. http://dx.doi.org/10.1016/j.chaos.2003.12.103

[14] Harjani, J., Rocha, J. and Sadarangani, K. (2015) Existence and Uniqueness of Solutions for a Class of Fractional Differential Coupled System with Integral Boundary Conditions. Applied Mathematics \& Information Sciences, 9, 401405.

[15] Le, M.H., Cordier, S., Lucas, C. and Cerdan, O. (2015) A Faster Numerical Scheme for a Coupled System Modeling Soil Erosion and Sediment Transport. Water Resources Research, 51, 987-1005. http://dx.doi.org/10.1002/2014WR015690

[16] Lu, M., Liu, B., Che, Y. and Han, C. (2015) Effect of Coupling Types on Synchronization of Weakly Coupled Bursting Neurons. 2015 International Symposium on Computers \& Informatics, Beijing, 17-18 January 2015, 1930-1937. http://dx.doi.org/10.2991/isci-15.2015.254

[17] Segall, K., Guo, S., Crotty, P., Schult, D. and Miller, M. (2014) Phase-Flip Bifurcation in a Coupled Josephson Junction Neuron System. Physica B: Condensed Matter, 455, 71-75. http://dx.doi.org/10.1016/j.physb.2014.07.048

[18] Zhang, Y., Zhang, C. and Zheng, B. (2011) Analysis of Bifurcation in a System of $n$ Coupled Oscillators with Delays. Applied Mathematical Modelling, 35, 903-914. http://dx.doi.org/10.1016/j.apm.2010.07.045

[19] Banerjee, T., Biswas, D. and Sarkar, B.C. (2012) Design and Analysis of a First Order Time-Delayed Chaotic System. Nonlinear Dynamics, 70, 721-734. http://dx.doi.org/10.1007/s11071-012-0490-3

[20] Banerjee, T., Biswas, D. and Sarkar, B.C. (2013) Anticipatory, Complete and Lag Synchronization of Chaos and Hyperchaos in a Nonlinear Delay-Coupled Time-Delayed System. Nonlinear Dynamics, 72, 321-332. http://dx.doi.org/10.1007/s11071-012-0490-3

[21] Wu, J. (1998) Symmetric Functional Differential Equations and Neural Networks with Memory. Transactions of the American Mathematical Society, 350, 4799-4838. http://dx.doi.org/10.1090/S0002-9947-98-02083-2

[22] Li, M.Y. and Muldowney, J.S. (1993) On Bendixson’s Criterion. Journal of Differential Equations, 106, 27-39. http://dx.doi.org/10.1006/jdeq.1993.1097 